\title{
A quality improvement management project to improve the management of ambulance inserted peripherally
} inserted cannulae (PIVC)

Need to remove or replace your patients ambulance intravenous cannule

\section{QAS-IVC}

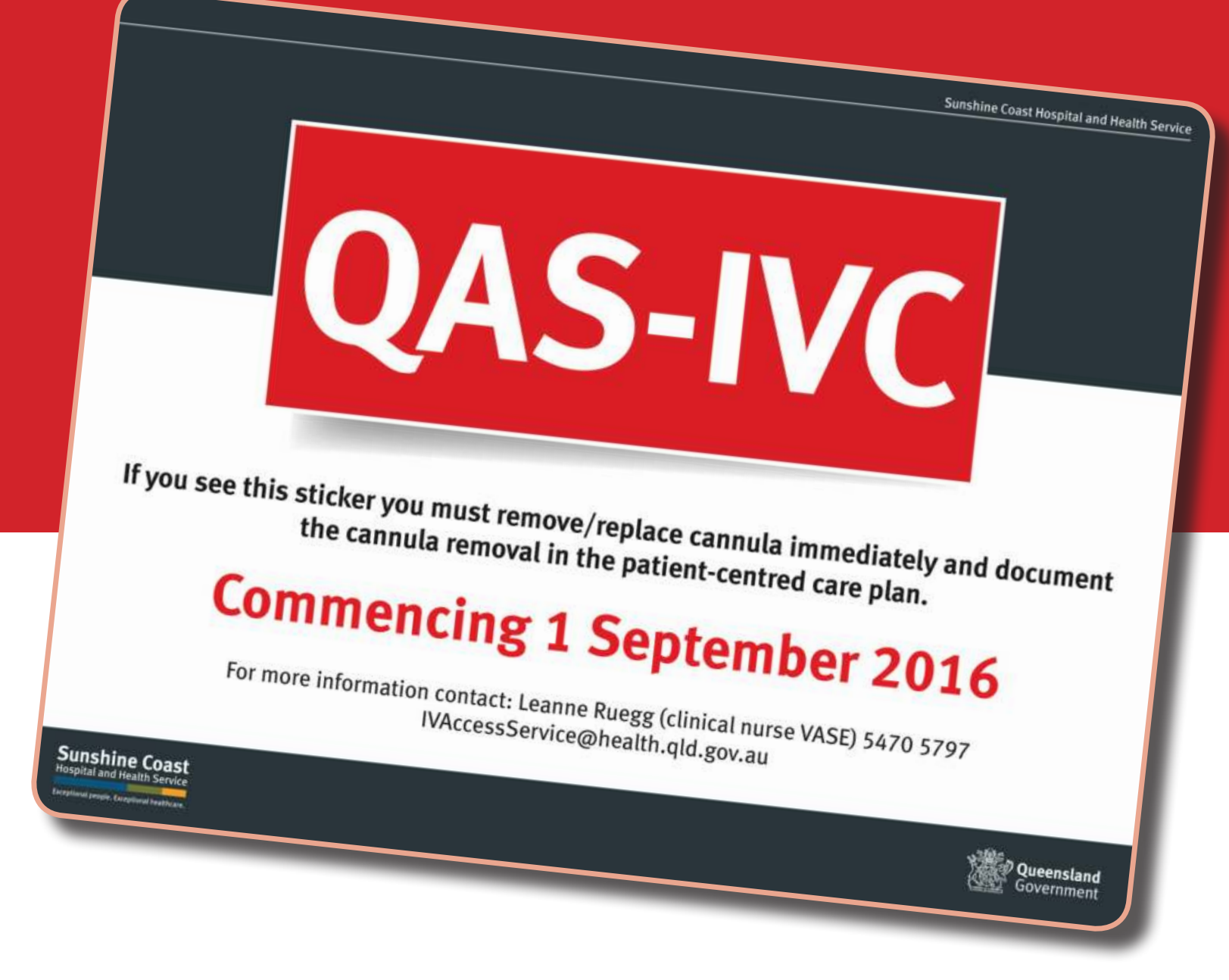

\section{Introduction}

Both the Sunshine Coast Hospital and Health Service (SCHHS) and Queensland Health Guidelines require PIVC inserted in emergency situations, when adherence to asepsis cannot be ensured, be replaced by a clinician within 24 hours or sooner if the patient's condition is stabilised. However despite the availability of the aforementioned guidelines, current audits performed by the SCHHS Vascular Access Surveillance and Education (VASE) team have shown that $73.7 \%$ of all ambulance inserted PIVC have a dwell time of greater than 24 hours despite our local procedure. The ability of health care workers to recognise ambulance inserted PIVC to facilitate timely removal was noted to be an issue hindering the timely removal.

There are close to 200,000 healthcare associated infections (HAls) in Australian acute facilities each year. Intravenous (IV) catheters cause over 3,500 cases of blood stream infections (BSIs) every year in Australia. BSI infections are associated with mortality of more than $10 \%$, and in many cases also significant morbidity. These events are potentially preventable through correct IVD management.

\section{Methods}

Pre-implementation data collected was date of audit and date of insertion, which professional stream inserted the PIVC, site assessment, documentation and dressing adequacy. Implementation of the quality improvement project consisted of stakeholder engagement, development and rollout of PIVC sticker to identify ambulance inserted PIVC and staff education. Post implementation data collection utilised the same audit tool as in preimplementation audit.

\section{Implementation of QI project}

- Stakeholder engagement, this included, meetings education resources for the Qld Ambulance service

- Staff education included presentations at relevant forums, screen saver on health service

intranet, this included both Medical and Nursing streams

- Development and roll out of PIVC sticker to identify ambulance inserted PIVC

\section{QAS-IVC}

- Staff education.

\section{Results}

The number of QAS cannulas present was reduced. SCHHS will be continuing with the sticke initiative. Feedback to clinical areas and QAS will continue to promote timely identification and removal of ambulance inserted PIVC.

\section{Conclusion}

The conclusion of the project was sticker identification prompted early removal of the PIVC within the recommended 24 hour timeframe from $26 \%$ pre-to $71 \%$ post. SCHHS will be continuing with the sticker initiative.
QAS PIVC 24 hour dwell time compliance

\section{$71 \%$}
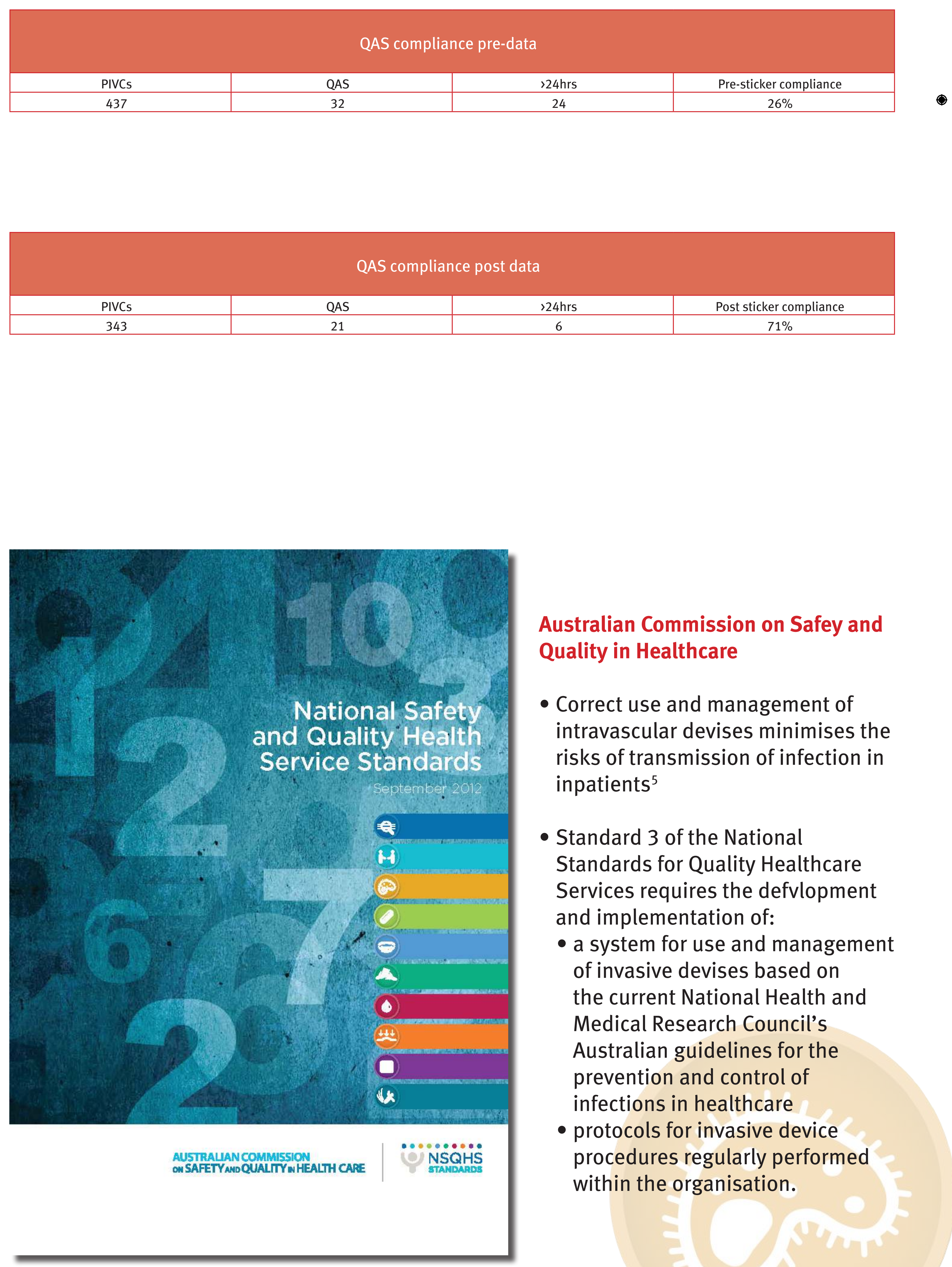

Australian Commission on Safey and Quality in Healthcare

- Correct use and management of intravascular devises minimises the risks of transmission of infection in inpatients

- Standard 3 of the National Standards for Quality Healthcare and implementation of: - a system for use and management of invasive devises based on the current National Health and Medical Researh Council's Medical Research Council's Australian guidelines for the prevention and control of infections in healthcare - protocols for invasive device procedures regularly performed within the organisation.

\section{Sunshine Coast} Hospital and Health Service 